\title{
Current Scenario and Future Direction of Newborn Screening and Management Program for Phenylketonuria in Bangladesh
}

Journal of Inborn Errors

of Metabolism \& Screening

2022, Volume 10: e20210024

DOI: https://doi.org/10.1590/2326-4594-

JIEMS-2021-0024

\author{
Abdul Alim Al-Bari' 1 (1)
}

\begin{abstract}
Phenylketonuria (PKU) is a correctable inborn error of metabolism which causes lethal intellectual delay and neurobehavioral anomalies. A screening package, especially for early recognition can support to regulate the PKU process of most patients. Newborn screening program in any country focuses at the earliest detection of inheritance deficiency disorders in order to avoid the most severe repercussion by appropriate medication. This screening program needs a concomitant diagnosis and involves additional clinical research. Strategies from developed countries recommend that new-born screening should be done as soon as possible after birth before hospital/clinic discharge because if detected later, it conveys to significantly increase in disability as well as morbidity. Although exact protocol differs among different countries, testing procedures for PKU should be followed universally recognized in the developed world. Unfortunately, new-born screening program in Bangladesh is in lying-in room or possibly in pilot study in particular hospital, because the health-care system is classically targeted mortality (like childbirth complications) and transmittable morbidities (such as COVID-19) but not inborn frailties. Although policies and management of childbirth complications have been successfully lowered infant and mother mortality rates, the number of disabled babies increased tremendously. The study aims to investigate the current status of new-born screening (NBS) program of PKU in the Rajshahi Division Bangladesh, and focus on future plans to manage with life-long treatment. The primary challenges such as financial support for newborn screening, publicity, should be identified and implemented for national PKU-NBS policy as a role model of Bangladesh for developing countries.
\end{abstract}

Keywords: Phenylketonuria, new-born screening, dried blood spot (DBS) cards, Guthrie test, Bangladesh.

\section{Introduction}

Alma-Ata Convention, 1978 declared that every child has identical right to acquire the basic healthcare facility regardless of sex, race/ethnic, society or nationality as a worldwide birth-right [1]. However, most of the developing countries have focused on quality of health services and abundant evidence proposes that quality of health care is almost absent in case of inherent newborn deficiency disorders [2-4]. Although new-born screening may turn to the earliest detection of inherent disorders, some screening test results have not been provided to advantage the baby being screened; false diagnostic results may create adverse consequences to the baby and its parents [5].

New-born screening goals to detect a designated number of hereditarily communicated diseases. Most of the cases, these disorders are autosomal recessive. Although these orders may pass through an altered gene expression, the parents do not typically display signs. For these reasons, there has not been any previous existence of the disease in the family history and the disorders happens unpredictably. New-born screening program has been considered to find out the infants with disease severity that are moderately predominant and curable or manageable [6,7]. Here it is reported that the initiation and society development of new-born screening programs have been developed in Bangladesh. Current challenges and limitations of the screening programs, expectations, and future directions are also highlighted.

\footnotetext{
${ }^{1}$ University of Rajshahi, Faculty of Science, Department of Pharmacy, Rajshahi-6205, Bangladesh.
}

Received May 26, 2021. Accepted for publication January 13, 2022.

\section{Corresponding Author:}

Abdul Alim Al-Bari, Email: alimalbari347@ru.ac.bd 


\section{World-Wide New-Born Screening Program}

For history, new-born screening was initially launched as a public health package in the USA in the early sixties, and has lengthened to different countries of the world, with different testing menus in each country [7]. Both prenatal screening (prebirth screening) and new-born screening (post birth screening) have assigned for improvement of health care system. PKU is the first disorder noticed by contemporary new-born screening programs. $\mathrm{PKU}$ is a metabolic condition in which the inability to degrade phenylalanine (Phe), an essential amino acid, results in irretrievable psychological retardation unless treated early. As a result, early detection and dietary treatment through new-born screening program, the negative impacts such as mental retardation of the disease can be largely eliminated $[8,9]$. For establishing new-born screening programs of PKU patients in the United Kingdom, Robert Guthrie developed a classical method for the detection of high levels of Phe in blood shortly after a baby born by using a bacterial inhibition assay [10]. Guthrie also established a simple analytical system for the collection of blood on filter paper which can be easily carried out and recognized the spot on a large scale and this method is increased popularity world-widely $[11,12]$. Later on, Guthrie test also allowed for the recognition of more metabolic disorders by evolving antibacterial tests for other metabolites e.g., leucine, an amino acid for maple syrup urine disease [11].

Phenylketonuria (PKU) is an autosomal recessive inborn disorder of phenylalanine (Phe) metabolism triggered by a deficit in the hepatic and renal enzyme L-Phe hydroxylase (PAH) [13]. Mutations in the $P A H$ gene cause in decreased catalytic activity that affects the catabolic pathway of phenylalanine (Phe) (Figure 1) and impairs the conversion of L-Phe to L-tyrosine (Tyr) resulting building up L-Phe in the body particularly blood and brain [14]. This accumulation of L-Phe eventually leads to serious health problems such as irreversible intellectual disability, motor deficits, seizures, autistic behaviors, eczematous rash and mental disorders. In some patients, persistently higher Phe levels can cause a range of comorbidities including neurocognitive, and neuropsychiatric. ${ }^{15} \mathrm{PAH}$ deficiency characterizes phenotypically as mild hyperphenylalaninaemia (MHP), mild PKU (mPKU), or classic PKU (cPKU) with the blood L-Phe level ranges $120-600 \mu_{\mathrm{moll}^{-1}}, 600-1200 \mathrm{moll}^{-1}$, $>1200 \mu_{\mathrm{moll}^{-1}}$ respectively.

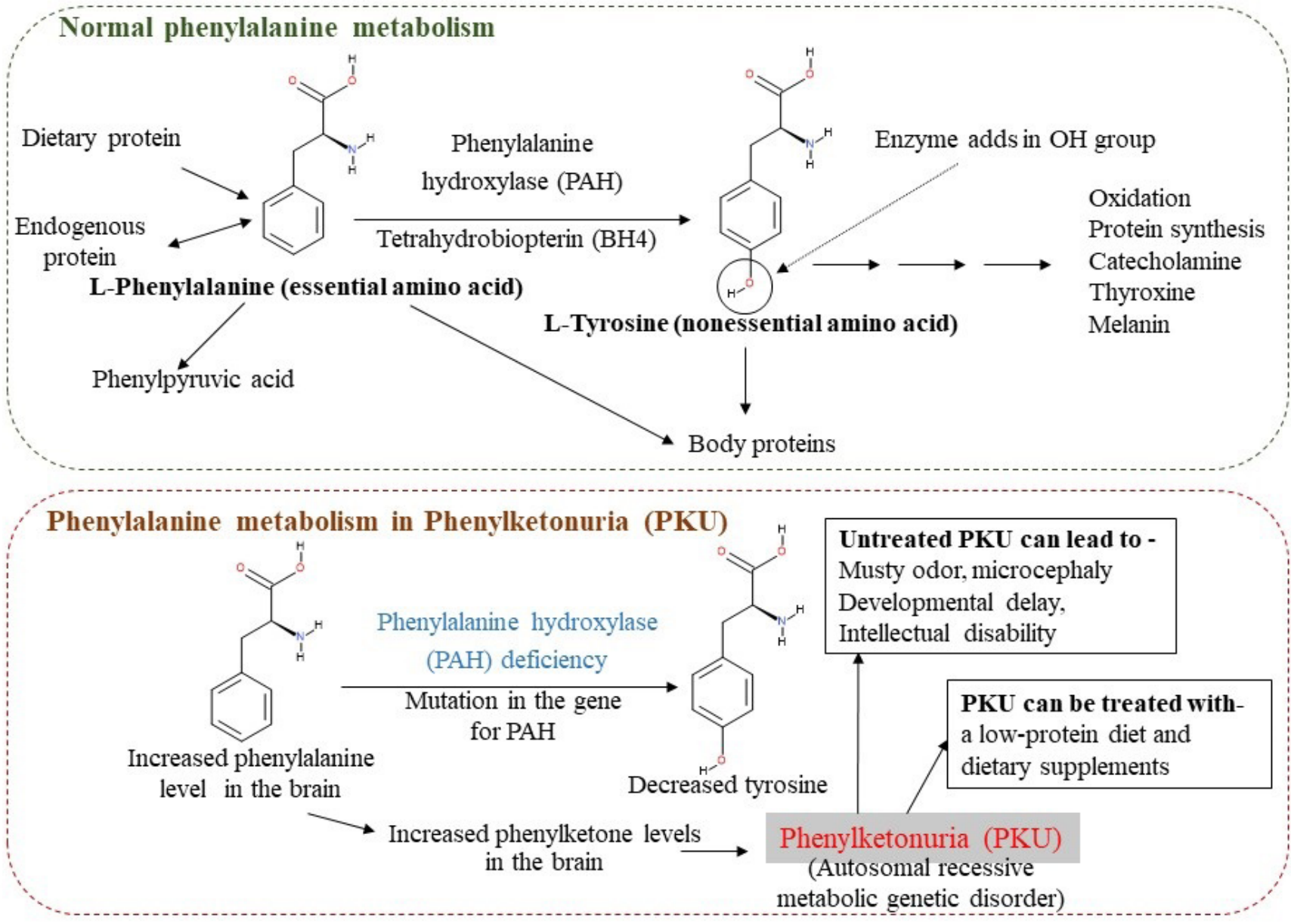

Figure 1. Inborn errors of phenylalanine metabolism: phenylketonuria (PKU). 
However, in case of $\mathrm{mPKU}$ and $\mathrm{cPKU}$, treatment is required. The frequency of PKU differs among racial groups and terrestrial regions in the world [16]. For example, in the USA the rate of incidence is 1:10,000 birth in Caucasians [17]. In Europe, the maximum occurrence has been detected in Ireland at a rate of 1:4,500. It is also found in several prefectures of China, while it is hardly detected in African countries. In Turkey, an occurrence as high as 1:2,600 has been described (the highest prevalence at $0.0167 \%)$. Moreover, the lowest prevalence is observed in the UAE, $0.005 \%$ [16]. This occurrence is higher for Caucasian and Native American inhabitants and fewer for African American, Hispanic and Asian people. The hepatic form of the enzyme $\mathrm{PAH}$ requires the cofactor tetrahydrobiopterin $\left(\mathrm{BH}_{4}\right)$, iron and oxygen for its complete activity in order to convert L-Phe to Tyr [18]. Since it can be regulated if treated shortly after birth with a low-Phe diet, a fast and accurate method for PKU diagnosis is urgently needed.

Like PKU, the thyroid hormone deficiency syndrome, congenital hypothyroidism $(\mathrm{CH})$ is also present at birth [19]. If untreated for several months after birth, severe $\mathrm{CH}$ leads to growth retardation and eternal intellectual disability. The hypothalamic-pituitary-thyroid axis starts functioning during mid-fetal life, and this activity continues till birth. If hypothyroidism is present in the embryo, it can lead to abnormalities in important organs such as central nervous system and skeleton. However, most infants with this disorder look completely healthy at birth. Therefore, given the effect of thyroid hormone on brain development, small and nonspecific symptoms of hypothyroidism in infants, and irreversible effects of this disorder, for many years hypothyroidism screening has been carried out in various countries [20].

\section{Obstacles of New-Born Screening Program in Bangladesh}

Interestingly, the most developed countries around the world carry out systematic screening programs for screening of PKU in infants shortly after birth. In Bangladesh, one group have started to diagnose of inborn errors of metabolism by using liquid chromatography tandem mass spectrometry (LC-MS/MS) in a pilot study [21]. However, the screening program should be covered throughout the country. Given the priority of higher birth rate and socioeconomical considerations in countries like Bangladesh, introducing new-born screening (NBS) program for these metabolic deficiency disorders would be a substantial but valuable challenge. The frequency, obscurity and varied scientific spectrum of inborn errors of metabolisms (IEMs) represent an intimidating diagnostic challenge to the researchers. However, in order to diminish morbidity and mortality, or other severe consequences like irretrievable neurological impairment to the patients with IEMs, early diagnosis and establishment of suitable therapy are very important. The practice of LC-MS/MS during the past eras has directed to an amazing upsurge in screening of IEMs. Many countries of the world have recognized NBS tests using LC-MS/MS which investigates metabolites from dried blood spots (DBSs) to distinguish the IEM-associated disorders, particularly the correctable one [22].

In Bangladesh, the scientific committee would be reformed by academicians, doctors, pharmacists, nurses and other healthcare professionals for screening of $\mathrm{PKU}, \mathrm{CH}$, biotinidase deficiency and other inherent diseases. In these ways, this program would be transformed into "national NBS program". This screening program would be implemented in various divisions including Rajshahi initially and then the national PKU screening program" covered throughout the country. Since this is a very large-scale program at the country level, the program would be faced several problems and limitations that necessitate changes in the screening process. The international organizational support for this program is urgently needed the policy making of this government to implement the development of mother and child health. International organization for preventive healthcare services and health protection can also promote the Government to initiate new-born screening program in Bangladesh.

The study would be reviewed and approved by the National Ethics Review Committee (NERC) of Bangladesh Medical Research Council (BMRC), Dhaka, Bangladesh. Prior to the collection of blood specimens, a written consent would be taken from the parents of all new born babies with IEMs and healthy participants. The whole blood specimens of newborns would be collected by heel prick method and spotted blood on Multipart filter paper to prepare a DBS (Dried blood spot) card for LC-MS/MS analysis [21] Specimen collection, storage, and preparation for LC-MS/MS Analysis need technical persons. Moreover, instrumentation and LC-MS/MS Analysis, data acquisition, data processing, validated and data acquisition by statistical analysis, all of these processes need several expert persons that are lack in Bangladesh. For these assistances, the international organization can support to conduct the screening and surveillance program and allocate financial supports for establishment of the central screening laboratory.

\section{Management Strategies for PKU}

Commencement of PKU treatment can be assured as soon as possible after detecting it, preferably within the first week of life. Depending on the detected blood Phe levels, this amino acid is essentially needed to exclude from the meal or a Pherestricted diet can be given. Suckling milk is frequently combined with therapeutic formula. Early commencement of treatment involves quick NBS program, follow-up, and investigative testing materials [23-25]. Sometimes the primary care providers can openly communicate with baby's family in order to access the appropriate specialty care.

In general, management of PKU comprises sudden lessening of dietetic total protein sources for decreasing the Phe intake [23]. Since Phe is an indispensable amino acid, total body growth cannot happen if it is entirely removed from the diet. The individual "tolerance" of Phe may differ in every child's 
due to representative level of enzymatic activity [23]. Thus, the consumed level of Phe in the diet must be assessed to the need of specifc child. This specific amount of Phe level can be attained by consuming measured quantities of PKU formula, which comprises altogether amino acids necessary for body growth excepting Phe, and ingesting of a diet with slight quantities of whole protein (which holds Phe). Classically, lactating or nourishing of steady formula will firstly be withheld for a PKU infant with a short duration (generally less than 3 days) in order to permit for rapid drop of Phe levels [26]. In this period the baby can drink only specific PKU formula deprived of Phe. When the levels started to fall, breastfeeding or typical newborn formula can be reinitiated in addition to PKU formula till Phe levels steady in the mark range. As PKU formula is fully Phe-free, PKU formula cannot use for prolonged periods of time such as longer than 1 to 2 days as a solitary nutrition source [27]. The Phe rations will modify as the child grows, and feeding sanctions will generally be provided by metabolic nutritionists skilled in PKU treatment [28].

There are different approaches of PKU therapy under progress. These therapeutics demonstrate the choice of solutions that can be organized to address the basic problem of PKU, the lack of catalytic action of the PAH enzyme. Although majority of these therapies have shown high efficacy in the animal models (such as murine) of PKU, all of the therapies need pharmacokinetic analysis and clinical trials to ensure the safety and longevity of treatment, as well as eliminating the rigorous dietary restrictions in case of patients with PKU [29]. These treatment strategies can also be used to other genetic metabolic disorders and this research into PKU treatments will have across-the-board significances in the field of genomic medicine in the next few years.

The matured new-born screening program supports in the initial founding of a Phe-restricted diet and the opportunity of circumventing brain damage in PKU patients. There are many concerns regarding current treatment strategies essential to be addressed and abundant efforts must be taken before these new therapies avail for patients [30]. The complications of sustaining a severe lifetime diet and the incidence of other difficulties (disdain treatment) make the search of novel healing tactics of countless importance. Although the launching of NBS programs in combination with quick foundation of nutritional action has prohibited intellectual disability, neurophysiological damages may still persevere in treated PKU patients $[26,31,32]$. Moreover, there are frequently difficulties associated with dietary treatment including nourishing deficiencies as well as non-compliance due to poor deliciousness [33-35]. Thus, the increasing information concerning about PKU pathogenesis has allowed the advance of several new therapeutic approaches. Therefore, advanced treatments will ultimately convert personalized therapy for addressing in patients with PKU on individual genotypes and other precise conditions. Molecular diagnosis by highthroughput sequencing techniques also supports for approval for laboratory-screening results of neonates and simplifies the detection of fresh genetic alterations connected to PKU [21]. Furthermore, the standard marketing pricing and precision of next generation sequencing (NGS) for molecular diagnostics [36] has instigated to display in substituting assenting biological screening for PKU in newborns in the near future.

The cofactor, tetrahydrobiopterin (BH4) for $\mathrm{PAH}$ has been effectively applied in $\mathrm{BH} 4$ sensitive patients; however, only a slight fraction of PKU patients get beneficial from this therapy [37,38]. Thus, several investigations on novel nutritional enhancements for the treatment of PKU is continuing such as including well-palatable therapeutic formulae, functional foods using glycomacropeptide (GMP), natural low-Phe protein and subjunction of large neutral amino acids (LNAA). Although LNAA supplement has been found to steady the cerebral Phe concentration, it causes an increase plasma Phe level, signifying that the LNAA influx may prevent Phe passage through the blood brain barrier (BBB). Moreover, although innovative therapeutic foods and formulas and improved palatability, including LNAA and GMP, have been explored [39-41] the chronic toxicological studies are urgently required to comprehend the complete impact of these treatments. Opposing data has been suggested that LNAA can act as a substitute to nutritional therapy and endorse for adult PKU patients. The combination of glycomacropeptide (GMP) into low Phe diet has enhanced palatability, diversity and suitability of the diet and has regulated blood Phe levels, suggesting a functional form of amino acid to be consumed by PKU patients [42] Although GMP may act as a promising amino acid supplement, long-term studies are desirable to evaluate the pharmacokinetic studies.

Fusion protein targeting enzyme replacement therapy into the liver has been auspicious in current years $[43,44]$ where Phe ammonia lyase (PAL) is applied as a substitute to PAH. Gene therapy have been progressing and PAL conjugated to polyethylene glycol (PEG) is being ongoing for phase III clinical trials. However, the efficiency of PAL is abridged over time and immune reactions are reported in some patients. Finally, probiotic generated PAL enzyme has increased consideration and clinical studies are ongoing $[45,46]$. These data acme the necessity to discovery alternate therapies for PKU that can be applied safely and efficiently in all PKU patients irrespective genotype, phenotype or sex differences.

\section{Special Care Considerations in PKU}

\section{Caring for Children with PKU}

PKU treatment comprises practically decrease of nutritional total protein sources for reducing the consumption of Phe. In most countries, nutritionists play an essential role in serving families for management of PKU diet. In this case blood Phe levels can be analyzed intermittently by getting a blood sample by heel or finger poke. 


\section{Advocacy of Family Physician}

Although PKU is a rare disorder, the family doctor can play a vivacious role in the establishment of consistent remedial care, nursing, harmonization of care, consultancy, and family provision. In most cases, children with PKU necessitate a slight expert medical care. However, special consciousness needs for specialized PKU management and family doctor can confidently deliver constant care. In this case, the most valuable resource persons can provide financial support the children with PKU and their families. The family doctor can influence and help children with PKU and their families for stunned substantial life challenges.

\section{Carbohydrate Status in Patients with PKU}

PKU patients are susceptible for intolerance of carbohydrate and insulin resistance particularly in elderly and obese patients. PKU patients who needed nutritional endorsements appear to have similar cardiovascular risks like the healthy people. However, other risk factors such as an atherosclerotic lipid profile, hypertension and C-reactive protein (CRP) levels have been stated in obese patients with PKU.

\section{Therapeutics and Multivitamin/Mineral Supplements}

Family doctors should be conscious about some food supplements such as artificial sweeteners that contain aspartame which may elevate Phe level. In case of severe or chronic illness, muscle wasting occurs and elevates Phe levels. Thus, special arrangement is needed in these cases. Further, special attention is needed to the female patients with PKU who is approaching to adolescence, contraceptive method and around pregnancy cycles. The pharmacist can also play a valuable role to manage PKU patients by checking the course of medication, recording frequency of administration or developing formulations. If a prescription comprises aspartame, the pharmacist can consult with guardian of PKU patient for providing an alternative medication. In addition, some multivitamin or mineral supplements also hold amino acids or skim milk powder acts as a source of Phe. The pharmacist can review new medications or supplements to determine whether the new preparation may act as a source of supplementary nutritional Phe intake.

\section{Mother Education}

Awareness and education of PKU for the mothers are major determinant of diet adherence and maintenance for the PKU baby patients because an excessively restraining PKU diet impairs total body growth in early childhood, probable due to undernourishment. These diets also contain low levels of longchain polyunsaturated fatty acids (LCPUFA) such as omega-3 fatty acid, for example docosahexaenoic acid (DHA), which are important for development of brain. Moreover, LCPUFA supplements has been found to improve brain development in children with PKU. Micronutrient shortage is also more common in children with PKU which leads to greater risk in these patients. Thus, consciousness and instruction of PKU for guardians particularly mothers are important for management of diets in PKU patients.

\section{Governmental Subsidies for Reimbursement of Treatment}

The expensive charge of special functional foods and therapeutic formula, as well as regular appointments to health professionals result in an extensive economic load on PKU patients and their families. The chief parts of PKU medical cost are the special therapeutic formula (low-protein foods) and supplements and annual out-of-pocket costs are the highest for (child $=\$ 1651$; adult $=\$ 967$ ) in USA. ${ }^{47}$ Furthermore, real dietetic agreement may be delayed by absence of health insurance or non-coverage of certain costs by insurance companies in some countries without a nationalized healthcare system. ${ }^{48}$ Although dietetic therapy may succeed the management of PKU patients, there is increasing data raising the issues of nutritional compliance and deficiencies. These results propose the urgently demand of novel therapeutic interventions that may progress the consequence for PKU patients. For reimbursement and social support, governments can provide financial support for treatment cost and contribute to deliver food supplements such as Phe-free L-amino acid. Patients at home countries should be disable entitled and government can provide a disability allowance and/or a disability certificate to cover out of package costs as well as social security of PKU patients.

\section{Conclusion and Future Perspective}

Genetic and congenital abnormalities in the first month of birth are the main causes of body organs defects and death in newborns. Every year, around 7.6 million children are born to the world with genetic or congenital abnormalities $90 \%$ of whom belong to countries with a low per capita income. The heavy burden caused by the neonatal diseases has made the states to plan and implement a screening program to diagnose neonatal and other types of metabolic diseases. The marriages taking place within immediate family relatives also increase the outbreak of the disease as well. The screening of new-borns at birth and the related healthcare sector are the tremendous burdens for a country; therefore, assessing the efficiency and effectiveness of the screening program in the healthcare sector as a health factor is essential. In order to enhance the quality of services and to economize, the organizations have to include the assessment process in their schedules and conduct the implementation in a scientific and technological manner. By implementing the screening program on new-borns throughout the nation, with the objective of accomplishing almost $100 \%$ coverage and completing the studies on other types of metabolic diseases together with adopting appropriate plans by determining the outbreak and related costs' level, it may be possible to incorporate the screening program of other diseases in the healthcare sector. 
Phenylketonuria (PKU) retards brain development and causes progressive mental impedance. The irreversible effects of this disease on the brain can be avoided if diagnosed and treated in the early days of life, before any clinical symptoms and signs appear, to prevent permanent damage to the very vulnerable neonatal brain. Thus, on-time diagnosis and a screening program followed by getting the treatment diet regime and controlling the Phe level in the blood are very important for PKU; otherwise, families would face heavy financial problems. Therefore, an analytical technique for fast and precise diagnosis of PKU is urgently desired. In order to achieve the objectives of this program and promote health in families, the accountability and high quality of the screening program must be proved. In this study, several attempts would be made to assess the different parts of the program in Rajshahi Division and apply the fundings in determining the in-born screening program and adopting policies to remove the miserable condition of health and promote the life.

Because of high rates of consanguinity in the areas of Bangladesh, genetic disorders may be more common. No estimates of the incidence or the actual percentage of new-borns screened for PKU in this area are available here. The inability to afford the costly diet of the PKU is a serious problem which many families of PKU new-borns have to face. Much of the governmental budget will need to cover some of the expenses of the treatment, rehabilitation, maintenance and special diet of the patients despite the shortage of resources in the healthcare sector, but initially we need to screening and discuss with Government for funding.

\section{Funding}

The author did not get any definite grants or funding for this effort.

\section{Ethics Approval and Consent to Participate}

MS is review based paper and not participating any humans or animals. Thus, human data or human tissue issue indicate "Not applicable"

\section{Declaration of Conflicting Interests}

The author declared no potential conflicts of interest with respect to the research, authorship, and/or publication of this article.

\section{References}

1. World Health Organization. Social determinants of health. WHO called to return to the Declaration of Alma-Ata. https://www.who.int/social_determinants/ tools/multimedia/alma_ata/en/. Accessed July 19, 2019.
2. Sarker SK, Islam MT, Biswas A, et al. Age-specific cut-off values of amino acids and acylcarnitines for diagnosis of inborn errors of metabolism using liquid chromatography tandem mass spectrometry. Biomed Res Int. 2019;2019:3460902. doi:10.1155/2019/3460902.

3. Mei L, Song P, Kokudo N, Xu L, Tang W. Current situation and prospects of newborn screening and treatment for Phenylketonuria in China - compared with the current situation in the United States, UK and Japan. Intractable Rare Dis Res. 2013;2(4):106-114. doi:10.5582/irdr.2013.v2.4.106.

4. Kickbusch I. Health promotion glossary WHO. Geneva, C.H.: Division of HPR, Health Education and Health Promotion Unit (HEP) WHO/HPR/HEP/98.1; 1998.

5. Zhu Z, Gu J, Genchev GZ, et al. Improving the diagnosis of phenylketonuria by using a machine learning-based screening model of neonatal MRM data. Front Mol Biosci. 2020;7:115. doi:10.3389/fmolb.2020.00115.

6. Tarini BA, Goldenberg AJ. Ethical issues with newborn screening in the genomics era. Annu Rev Genomics Hum Genet. 2012;13:381-393. doi:10.1146/annurevgenom-090711-163741.

7. Wieser B. Public accountability of newborn screening: collective knowing and deciding. Soc Sci Med. 2010;70(6):926-933. doi:10.1016/j.socscimed.2009.12.001.

8. Kreile M, Lubina O, Ozola-Zalite I, et al. Phenylketonuria in the Latvian population: molecular basis, phenylalanine levels, and patient compliance. Mol Genet Metab Rep. 2020;25:100671. doi:10.1016/j.ymgmr.2020.100671.

9. Kronn D. Navigating newborn screening in the NICU: a user's guide. Neoreviews. 2019;20(5):e280-e291. doi:10.1542/ neo.20-5-e280.

10. Gregory CO, Yu C, Singh RH. Blood phenylalanine monitoring for dietary compliance among patients with phenylketonuria: comparison of methods. Genet Med. 2007;9(11):761-765. doi:10.1097/GIM.0b013e318159a355.

11. Groselj U, Tansek MZ, Battelino T. Fifty years of phenylketonuria newborn screening - A great success for many, but what about the rest? Mol Genet Metab. 2014;113(1-2):8-10. doi:10.1016/j.ymgme.2014.07.019.

12. Lopes MEM. The successful "Guthrie test" celebrates its 10th birthday in Brazil! Ciên Saúde Colet. 2011;16 Suppl 1:716-717. doi:10.1590/s1413-81232011000700001.

13. Scriver CR. The PAH gene, phenylketonuria, and a paradigm shift. Hum Mutat. 2007;28(9):831-845. doi:10.1002/ humu.20526.

14. Scriver CC. A simple phenylalanine method for detecting phenylketonuria in large populations of newborn infants, by Robert Guthrie and Ada Susi, Pediatrics, 1963;32:318343. Pediatrics. 1998;102(1 Pt 2):236-237. 
15. Therrell Jr BL, Lloyd-Puryear MA, Camp KM, Mann MY. Inborn errors of metabolism identified via newborn screening: ten-year incidence data and costs of nutritional interventions for research agenda planning. Mol Genet Metab. 2014;113(1-2):14-26. doi:10.1016/j.ymgme.2014.07.009.

16. El-Metwally A, Al-Ahaidib LY, Sunqurah AA, et al. The prevalence of Phenylketonuria in Arab Countries, Turkey, and Iran: a systematic review. Biomed Res Int. 2018;2018:7697210. doi:10.1155/2018/7697210.

17. Williams RA, Mamotte CDS, Burnett JR. Phenylketonuria: an inborn error of phenylalanine metabolism. Clin Biochem Rev. 2008;29(1):31-41.

18. Blau N, van Spronsen FJ, Levy HL. Phenylketonuria. Lancet. 2010;376(9750):1417-1427. doi:10.1016/S01406736(10)60961-0.

19. Caiulo S, Corbetta C, Di Frenna M, et al. Newborn screening for congenital hypothyroidism: the benefit of using differential TSH cutoffs in a two-screen program. J Clin Endocrinol Metab. 2021;106(1):e338-e349. doi:10.1210/ clinem/dgaa789.

20. Brown RS, Demmer LA. The etiology of thyroid dysgenesisstill an enigma after all these years. J Clin Endocrinol Metab. 2002;87(9):4069-4071. doi:10.1210/jc.2002-021092.

21. Sarker SK, Islam MT, Biswas A, et al. Age-specific cut-off values of amino acids and acylcarnitines for diagnosis of inborn errors of metabolism using liquid chromatography tandem mass spectrometry. Biomed Res Int. 2019;2019:3460902. doi:10.1155/2019/3460902.

22. Millington DS, Kodo N, Norwood DL, Roe CR. Tandem mass spectrometry: a new method for acylcarnitine profiling with potential for neonatal screening for inborn errors of metabolism. J Inherit Metab Dis. 1990;13(3):321324. doi:10.1007/BF01799385.

23. Macdonald A, Daly A, Davies P, et al. Protein substitutes for PKU: what's new? J Inherit Metab Dis. 2004;27(3):363371. doi:10.1023/B:BOLI.0000031099.79046.65.

24. Sumaily KM, Mujamammi AH. Phenylketonuria: a new look at an old topic, advances in laboratory diagnosis, and therapeutic strategies. Int J Health Sci (Qassim). 2017;11(5):63-70.

25. Collins JE, Leonard JV. The dietary management of inborn errors of metabolism. Hum Nutr Appl Nutr. 1985;39(4): $255-272$.

26. Channon S, Mockler C, Lee P. Executive functioning and speed of processing in phenylketonuria. Neuropsychology. 2005;19(5):679-686. doi:10.1037/0894-4105.19.5.679.

27. Baulny HO, Abadie V, Feillet F, de Parscau L. Management of phenylketonuria and hyperphenylalaninemia. $J$ Nutr. 2007;137(6 Suppl 1):1561S-1563S. doi:10.1093/ jn/137.6.1561S.
28. Brumm VL, Grant ML. The role of intelligence in phenylketonuria: a review of research and management. Mol Genet Metab. 2010;99 Suppl 1:S18-21. doi:10.1016/j. ymgme.2009.10.015.

29. Feillet F, Clarke L, Meli C, et al. Pharmacokinetics of sapropterin in patients with phenylketonuria. Clin Pharmacokinet. 2008;47(12):817-825. doi:10.2165/0003088200847120-00006.

30. van Spronsen FJ, Enns GM. Future treatment strategies in phenylketonuria. Mol Genet Metab. 2010;99 Suppl 1:S9095. doi:10.1016/j.ymgme.2009.10.008.

31. Huijbregts SCJ, de Sonneville LMJ, Licht R, van Spronsen FJ, Verkerk PH, Sergeant JA. Sustained attention and inhibition of cognitive interference in treated phenylketonuria: associations with concurrent and lifetime phenylalanine concentrations. Neuropsychologia. 2002;40(1):7-15. doi:10.1016/s0028-3932(01)00078-1.

32. Huijbregts SCJ, de Sonneville LMJ, van Spronsen FJ, Licht $\mathrm{R}$, Sergeant JA. The neuropsychological profile of early and continuously treated phenylketonuria: orienting, vigilance, and maintenance versus manipulation-functions of working memory. Neurosci Biobehav Rev. 2002;26(6):697-712. doi:10.1016/s0149-7634(02)00040-4.

33. MacDonald A, Gokmen-Ozel H, van Rijn M, Burgard P. The reality of dietary compliance in the management of phenylketonuria. J Inherit Metab Dis. 2010;33(6):665-670. doi:10.1007/s10545-010-9073-y.

34. Acosta PB, Yannicelli S, Singh R, et al. Intake and blood levels of fatty acids in treated patients with phenylketonuria. J Pediatr Gastroenterol Nutr. 2001;33(3):253-259. doi:10.1097/00005176-200109000-00005.

35. Acosta PB, Yannicelli S, Singh R, et al. Nutrient intakes and physical growth of children with phenylketonuria undergoing nutrition therapy. J Am Diet Assoc. 2003;103(9):1167-1173. doi:10.1016/S00028223(03)00983-0.

36. Lampret BR, Remec ŽI, Torkar AD, et al. Expanded newborn screening program in Slovenia using tandem mass spectrometry and confirmatory next generation sequencing genetic testing. Zdr Varst. 2020;59(4):256263. doi:10.2478/sjph-2020-0032.

37. Burnett JR. Sapropterin dihydrochloride (Kuvan/ phenoptin), an orally active synthetic form of $\mathrm{BH} 4$ for the treatment of phenylketonuria. IDrugs. 2007;10(11): 805-813.

38. Acosta PB, Yannicelli S, Singh RH, Elsas 2nd LJ, Mofidi S, Steiner RD. Iron status of children with phenylketonuria undergoing nutrition therapy assessed by transferrin receptors. Genet Med. 2004;6(2):96-101. doi:10.1097/01. gim.0000117335.50541.f3. 
39. Ney DM, Gleason ST, van Calcar SC, et al. Nutritional management of PKU with glycomacropeptide from cheese whey. J Inherit Metab Dis. 200932(1):32-39. doi:10.1007/ s10545-008-0952-4.

40. Solverson P, Murali SG, Litscher SJ, Blank RD, Ney DM. Low bone strength is a manifestation of phenylketonuria in mice and is attenuated by a glycomacropeptide diet. PLoS One. 2012;7(9):e45165. doi:10.1371/journal.pone.0045165.

41. van Calcar SC, MacLeod EL, Gleason ST, et al. Improved nutritional management of phenylketonuria by using a diet containing glycomacropeptide compared with amino acids. Am J Clin Nutr. 2009;89(4):1068-1077. doi:10.3945/ ajcn.2008.27280.

42. Daly A, Evans S, Pinto A, et al. The impact of the use of glycomacropeptide on satiety and dietary intake in phenylketonuria. Nutrients. 2020;12(9):2704. doi:10.3390/ nu12092704.

43. Viecelli HM, Harbottle RP, Wong SP, et al. Treatment of phenylketonuria using minicircle-based naked-DNA gene transfer to murine liver. Hepatology. 2014;60(3):1035-1043. doi:10.1002/hep.27104.
44. Yagi H, Ogura T, Mizukami H, et al. Complete restoration of phenylalanine oxidation in phenylketonuria mouse by a self-complementary adeno-associated virus vector. J Gene Med. 2011;13(2):114-122. doi:10.1002/jgm.1543.

45. Steidler L, Hans W, Schotte L, et al. Treatment of murine colitis by Lactococcus lactis secreting interleukin-10. Science. 2000;289(5483):1352-1355. doi:10.1126/science.289.5483.

46. de LeBlanc AM, LeBlanc JG, Perdigón G, et al. Oral administration of a catalase-producing Lactococcus lactis can prevent a chemically induced colon cancer in mice. J Med Microbiol. 2008;57(Pt 1):100-105. doi:10.1099/ jmm.0.47403-0.

47. Rose AM, Grosse SD, Garcia SP, et al. The financial and time burden associated with phenylketonuria treatment in the United States. Mol Genet Metab Rep. 2019;21:100523. doi:10.1016/j.ymgmr.2019.100523.

48. Guest JF, Bai JJ, Taylor RR, Sladkevicius E, Lee PJ, Lachmann RH. Costs and outcomes over 36 years of patients with phenylketonuria who do and do not remain on a phenylalanine-restricted diet. J Intellect Disabil Res. 2013;57(6):567-579. doi:10.1111/j.1365-2788.2012.01568.x. 